\title{
Characterization of a Novel DWD Protein that Participates in Heat Stress Response in Arabidopsis
}

\author{
Soon-Hee Kim ${ }^{1,4}$, Joon-Hyun Lee ${ }^{1,4}$, Kyoung-In Seo ${ }^{1,2}$, Boyeong Ryu ${ }^{1}$, Yongju Sung ${ }^{1}$, \\ Taijoon Chung ${ }^{3}$, Xing Wang Deng ${ }^{2}$, and Jae-Hoon Lee ${ }^{1,2, *}$
}

\begin{abstract}
Cullin4-RING ubiquitin ligase (CRL4) is a family of multisubunit E3 ligases. To investigate the possible involvement of CRL4 in heat stress response, we screened T-DNA insertion mutants of putative CRL4 substrate receptors that exhibited altered patterns in response to heat stress. One of the mutants exhibited heat stress tolerance and was named heat stress tolerant DWD1 (htd1). Introduction of HTD1 gene into htd1-1 led to recovery of heat sensitivity to the wild type level, confirming that the decrease of HTD1 transcripts resulted in heat tolerance. Therefore, HTD1 plays a negative role in thermotolerance in Arabidopsis. Additionally, HTD1 directly interacted with DDB1a in yeast two-hybrid assays and associated with DDB1b in vivo, supporting that it could be a part of a CRL4 complex. Various heat-inducible genes such as HSP14.7, HSP21, At2g03020 and WRKY28 were hyper-induced in htd1-1, indicating that HTD1 could function as a negative regulator for the expression of such genes and that these genes might contribute to thermotolerance of $h t d 1-1$, at least in part. HTD1 was associated with HSP90-1, a crucial regulator of thermotolerance, in vivo, even though the decrease of HTD1 did not affect the accumulation pattern of HSP901 in Arabidopsis. These findings indicate that a negative role of HTD1 in thermotolerance might be achieved through its association with HSP90-1, possibly by disturbing the action of HSP90-1, not by the degradation of HSP90-1. This study will serve as an important step toward understanding of the functional connection between CRL4-mediated processes and plant heat stress signaling.
\end{abstract}

\footnotetext{
${ }^{1}$ Department of Biology Education, Pusan National University, Busan 609-735, Korea, ${ }^{2}$ Department of Molecular, Cellular and Developmental Biology, Yale University, New Haven, Connecticut 06520-8104, USA, ${ }^{3}$ Department of Biological Sciences, Pusan National University, Busan 609-735, Korea, ${ }^{4}$ These authors contributed equally to this work.

*Correspondence: jhlee72@pusan.ac.kr

Received 6 August, 2014; revised 2 September, 2014; accepted 3 September, 2014; published online 2 October, 2014
}

\section{INTRODUCTION}

Plants are subject to a variety of abiotic stresses such as high/low temperature, wound, UV, water deficit and high salts (Bohnert et al., 2006). Although heat (high temperature) stress is becoming one of the most threatening abiotic stresses, it is still not clear how plants cope with this stress (Baniwal et al., 2004). The greenhouse effect is increasing the temperature of biosphere, which has a detrimental effects on the metabolism of living organisms. In the case of plants, a slight elevation in temperature adversely affects crop productivity (Mittler and Blumwald, 2010). Heat stress leads to negative effects on a variety of cellular events including loss of membrane integrity, accumulation of reactive oxygen species, cytoskeletal perturbation, and destruction of RNA and proteins (Kampinga et al., 1995; Ruelland and Zachowski, 2010; Vierling, 1991). To overcome the damage caused by heat stress, plants have developed exquisite mechanisms for thermotolerance in which various signaling components such as protein kinases, transcription factors and chaperones participate. Among them, heat shock proteins (HSP) and heat stress factors (Hsf) play a pivotal role in heat-tolerance (Todaka et al., 2012). Heat shock proteins were initially identified as proteins induced in response to high temperature. Following heat stress, HSP101, HSP90, HSP70, HSP60, HSP40 and SHSP (small HSP) associates with unfolded proteins caused by the stress, preventing their aggregation and facilitating proper refolding, which leads to their functional recovery (Baniwal et al., 2004; Boston et al., 1996). In addition to the contribution heat shock proteins play in survival under heat stress, they also play an important role in plant growth under normal and abiotic stress conditions (Wang et al., 2004). HSP90 has ATP-dependent hydrolysis activity and forms multichaperone complexes with other proteins such as HSP70, HSP70 interacting protein (Hip), HSP70/HSP90 organizing protein (Hop), HSP40 and p23 to enable its function as a chaperone (Wang et al., 2004). In cells, HSP90 are involved in diverse cellular processes such as maturation of signaling components, regulation of protein stability and trafficking, and control of the cell cycle (Richter and Buchner, 2001). In Arabidopsis, seven HSP90 proteins have been reported. The expression of Arabidopsis HSP9O genes are largely affected by cold, salt and heavy metal stresses, as well as heat stress,

Keywords: Arabidopsis, CRL4, heat stress, HSP, HTD1 
implying their complex involvement in abiotic stress signaling (Krishna and Gloor, 2001). Among HSP90 members, AtHSP90-1 is known to be the strongest heat-inducible cytosolic form and to play a major role in thermotolerance (Yabe et al., 1994). One of the most-actively studied proteins involved in heat response is AtHSP101 (also known as cytosolic ClpB1), which is necessary for acclimation to heat stress (Hong and Vierling, 2001). HSP101 is an AAA+ chaperone ATPase that performs ATP-dependent dissolution of protein aggregates with the sHsp chaperone system to enable recovery of proteins damaged by heat stress (Lee et al., 2005; Neuwald et al., 1999). Another crucial member, the HSP70 family, has been reported to participate in thermotolerance and resistance against abiotic stresses in plants (Lee and Schöffl, 1996; Ono et al., 2001; Sugino et al., 1999; Sung et al., 2001). Heat stress transcription factors (Hsfs) are key regulators involved in the expression of various heat-shock induced genes, including HSPs. All plant Hsfs commonly possess nuclear localization signal (NLS), DNA binding domains and the oligomerization domain (HR-A/B region). Hsfs use heat stress elements in the promoters of heatresponsive genes as cis-acting elements for gene expression (von Koskull-Döring et al., 2007). Although the master regulator of heat response in Arabidopsis is still elusive, HsfA2 appears to be the dominant form for thermotolerance response since HsfA2 accumulates during heat stress/recovery cycles and its loss leads to marked alteration of the expression of heat-shock responsive genes following heat treatments (Schramm et al., 2006).

Ubiquitination, a representative post-translational modification by eukaryotes, plays an important role in the regulation of protein stability, enzyme activity, and the cellular localization of proteins. Especially, the chain formation of ubiquitins into substrate functions as a tag to degrade substrate via $26 \mathrm{~S}$ proteasome complex. In plants, this process is involved in a variety of cellular events including biotic and abiotic stress responses, cell cycle progression and hormone responses (Smalle and Vierstra, 2004; Vierstra, 2009). Ubiquitination sequentially proceeds with three kinds of enzymes: E1 as an ubiquitinactivating enzyme, E2 as an ubiquitin-conjugating enzyme, and E3 as an ubiquitin ligase (Hershko and Ciechanover, 1998). When compared with the numbers of E1 and E2 (6 E1 and 49 E2), Arabidopsis has many more E3 ligases (1300 E3) in its genome, suggesting the importance of E3 in recognition of substrate (Dreher and Callis, 2007; Vierstra, 2009). Cullin4RING ubiquitin ligase (CRL4) is a type of cullin-RING E3 ligase (CRL) that acts as a multi-subunit E3 ligase complex. CRL4 is composed of RBX1 as a RING finger domain protein for E2 recruitment of DAMAGED DNA BINDING 1 (DDB1) as an adaptor from the complex, and DDB1 BINDING WD40 (DWD)/WDxR protein as a substrate receptor (Angers et al., 2006; Biedermann and Hellmann, 2010; He et al., 2006; Higa et al., 2006; Jin et al., 2006; Vierstra, 2009). DWD proteins commonly possess the DWD domain, which consists of 16 conserved amino acids within WD40 repeats, and 85 DWD proteins have been reported to exist in Arabidopsis (Lee et al., 2008). The following research for the substrate receptor of CRL4 in Arabidopsis suggested that an additional 34 proteins (WDxR) that only contain a portion of the DWD domain (WDxR region) also act as substrate receptors for CRL4 complex (Zhang et al., 2008). Based on these reports, it is believed that Arabidopsis have 119 potential substrate receptors for CRL4. There have been several studies of the functional involvement of DWDMDxR in plant abiotic stress signaling. Two DWD proteins, DWD hypersensitive to ABA (DWA) 1 and DWA2, have been reported as negative regulators in ABA-mediated drought stress signaling (Lee et al., 2010). dwa1dwa2 double mutants exhibit marked hypersensitivities in response to $A B A$ and salt stress, and drought tolerance. Moreover, DWA1 and DWA2 are responsible for $A B \mid 5$ degradation via direct interaction with $A B I 5$, a key regulator in $A B A$ signaling. Recently, a WDXR protein, ABA-HYPERSENSITIVE DCAF1 (ABD1), was identified as another negative regulator in $\mathrm{ABA}$-mediated drought tolerance (Seo et al., 2014). UV-B signaling is also mediated by a group of DWD proteins such as COCKAYNE SYNDROME A-LIKE PROTEINS 1A (CSAat1A, also known as ATCSA-1), CSAat1B, and DAMAGE-SPECIFIC DNA BINDING PROTEIN 2 (DDB2), which are reportedly responsible for UV-B-induced damage repair (Biedermann and Hellmann, 2010; Zhang et al., 2010).

Despite emerging efforts to elucidate the functional relationship between abiotic stress signaling and CRL-mediated process in plants, the role of CRL in heat stress tolerance is poorly understood. Here, we report on heat stress to lerant DWD1 (HTD1), a novel DWD that functions as a negative regulator in thermotolerance.

\section{MATERIALS AND METHODS}

Plant materials and growth conditions

Arabidopsis thaliana ecotype Columbia- 0 was used as the wild type in this study. htd1-1 (SALK_081295) was obtained from the Arabidopsis Stock Center (http://www.arabidopsis.org/). Arabidopsis seedlings were grown as previously described (Lee et al., 2008) on GM plates or soils under long-day conditions (16 h light/8 h dark) in a controlled-environmental chamber at $22^{\circ} \mathrm{C}$.

\section{Heat stress treatment}

Heat stress treatment was performed as described by Meiri and Breiman (2009), with slight modification. Briefly, three day-old wild type and $h t d 1-1$ seedlings cultivated at $22^{\circ} \mathrm{C}$ were incubated at $37^{\circ} \mathrm{C}$ for $3 \mathrm{~h}$ for acclimation, then allowed 3 days of recovery at $22^{\circ} \mathrm{C}$. Next, heat shock treatment at $45^{\circ} \mathrm{C}$ was applied for $90 \mathrm{~min}$, after which seedlings were grown for an additional 3 days to monitor phenotypes or conduct quantitative real-time PCR (qPCR) and inmmunoblot analysis.

Genomic DNA extraction and genotyping analysis Genomic DNA was extracted with Edwards buffer $(200 \mathrm{mM}$ Tris- $\mathrm{HCl}, \mathrm{pH}$ 7.5, $250 \mathrm{mM} \mathrm{NaCl}, 25 \mathrm{mM}$ EDTA and 0.5\% SDS) and then used for PCR analysis with LBb1.3 primer and the forward and reverse primers for HTD1. The DNA primer sequences used for genotyping analysis are listed in Supplementary Table S1.

RNA isolation and semi-quantitative RT-PCR analysis Total RNA was obtained using PureLink ${ }^{T M}$ RNA mini kits (Ambion). Semi-quantitative RT-PCR analyses were performed as previously described (Lee et al., 2008). Amplicons produced with RPN6a_Forward Primer and RPN6a Reverse Primer were used as internal controls. DNA sequences for the primer pairs used in semi-quantitative RT-PCR are presented in Supplementary Table S1.

\section{Quantitative real-time PCR (qPCR) analysis}

Quantitative PCR assay was conducted using a Solg ${ }^{\text {TM }} 2 \times$ Real-Time PCR Smart mix (SolGent) according to the manufacturer's instructions and the Rotor-Gene $Q$ system (Qiagen). Transcript amounts of various genes from each sample were obtained using the comparative CT method and normalized against ACTIN2 expression. All assays were performed in- 
A

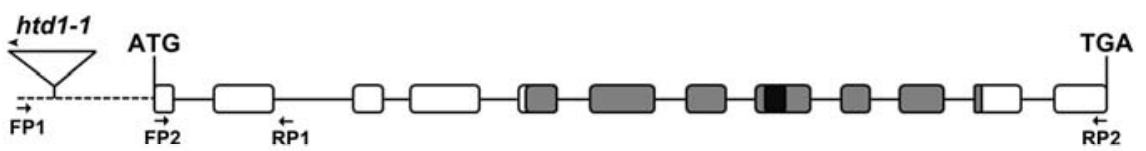

B

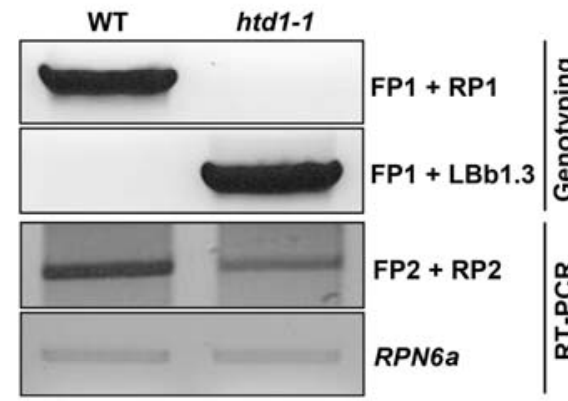

C

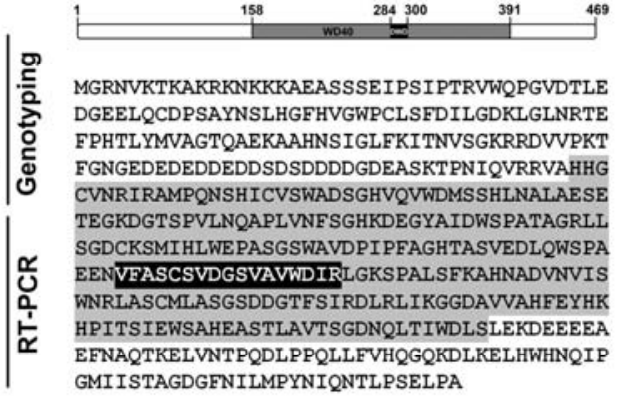

D
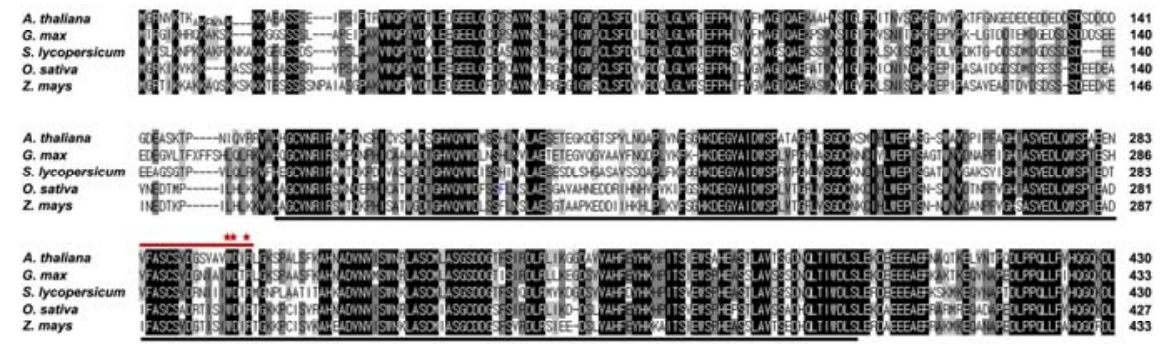

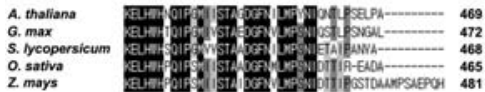

Fig. 1. Characterization of $h t d 1-1$ and HTD1. (A) Genomic structure and TDNA insertions in HTD1 and HTD1 protein structure. Exons are shown as boxes, introns are represented by lines and the $5^{\prime}$-untranslated region is depicted by dotted lines. Grey and black boxes indicate the WD40 region and DWD domain, respectively. The arrows indicate the position and direction of primers for genotyping and RT-PCR analysis. The arrowhead represents the direction of the LBb1.3 primer for genotyping analysis. (B) Genotyping and semi-quantitative RT-PCR analysis of wild type and htd1-1. RPN6a was used an internal control for semiquantitative RT-PCR assays. (C) Amino acid sequence of HTD1. The gray-shaded region represents the WD40 repeats and the black-shaded region indicates the DWD domain. (D) Alignment of the HTD1 protein and their homologues in various plant species. The alignment was performed with HTD1 and the proteins that showed the highest homology with HTD1 from each species. The corresponding proteins are A. thaliana HTD1 (NP_179544), G. max glutamate-rich WD repeat-containing protein 1-like (XP_003531198), S. lycopersicum glutamate-rich WD repeatcontaining protein 1-like isoform 2 (XP_004243762), O. sativa Os11g013

4500 (NP_001065677) and Z. mays LOC100217083 (NP_001136925). The WD40 domains are underlined with black lines and the DWD domains with a red line. The red asterisks represent the conserved tryptophan, aspartate and arginine residues with the WDxR motif. The regions with a high-level of conservation are indicated with black shade (100\%), a middle-level with dark gray $(80 \%)$ and a low level with light gray $(60 \%)$.

dependently at least three times. DNA sequences for the primer pairs used in qPCR are presented in Supplementary Table S2.

Generation of the 35S:HTD1/htd1-1 transgenic lines and the HTD1-MYCIFLAG-DDB1b/ddb1a transgenic plants For the 35S:HTD1/htd1-1 transgenic lines, the entire coding region for HTD1 was introduced into the binary vector pJIM19 (Gentamycin) under control of the cauliflower mosaic virus $35 \mathrm{~S}$ promoter (Lee et al., 2010). The generated construct was then introduced into Agrobacterium strain GV3101 by electroporation and into Col-0 plants by the floral-dip method (Clough and Bent, 1998). The resulting T1 seeds were selected on MS plates containing $200 \mathrm{mg} \mathrm{L}^{-1}$ gentamycin to obtain T1 transgenic lines (Clough and Bent, 1998). In the case of HTD1MYC/FLAG-DDB1b/ddb1a transgenic plants, the coding region of HTD1 cDNA was fused in frame to the $5^{\prime}$ end of nine copies of MYC that had already been subcloned in pJIM19 (Gentamycin) under control of the cauliflower mosaic virus $35 \mathrm{~S}$ promoter. Finally, the generated construct was transformed into FLAGDDB1b/ddb1a transgenic plants (Lee et al., 2008) via the GV3101 strain.
Protein isolation and Western blot analysis

Protein isolation and Western blot analysis were performed as previously described (Lee et al., 2010). Arabidopsis samples were extracted with $50 \mathrm{mM}$ Tris- $\mathrm{HCl}, \mathrm{pH} 7.5,150 \mathrm{mM} \mathrm{NaCl}, 0.1 \%$ Tween 20, 10\% glycerol, 1 mM DTT, 1 mM phenylmethylsulfonyl fluoride and $1 \times$ complete protease inhibitor (Roche). Extracted proteins were fractioned by SDS-PAGE and transferred to nitrocellulose membranes. The resulting blot was incubated in blocking buffer (1× PBS buffer including $0.1 \%$ Tween 20 and $5 \%$ dried nonfat milk) for $1 \mathrm{~h}$ at room temperature. Membranes were then incubated with the appropriate primary antibody for $2 \mathrm{~h}$ at room temperature or overnight at $4^{\circ} \mathrm{C}$. Next, samples were incubated with secondary antibodies (horseradish peroxidase-conjugated anti-rabbit or anti-mouse $\operatorname{lgG}$ ) for $1 \mathrm{~h}$ at room temperature. Proteins were then visualized using a western blotting detection system (EZ-Capture II, ATTO). The assays were performed with anti-FLAG (Sigma-Aldrich), anti-MYC (Cell Signaling), antiRPN6 (Chen et al., 2006), anti-HSP90-1 (Agrisera), anti-HSP70 (Agrisera), anti-HSP101 (Antibodies) or anti- $\alpha$-tubulin (SigmaAldrich).

Yeast two-hybrid assay

Yeast two-hybrid assays were performed according to the 
A Possible CRL4 Involved in Heat Stress Response

Soon-Hee Kim et al.

A

(-) Heat Shock

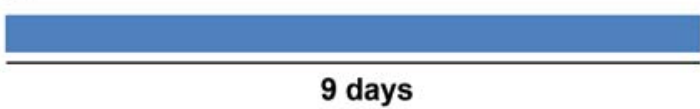

(+) Heat Shock

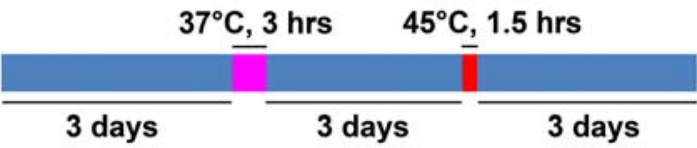

B
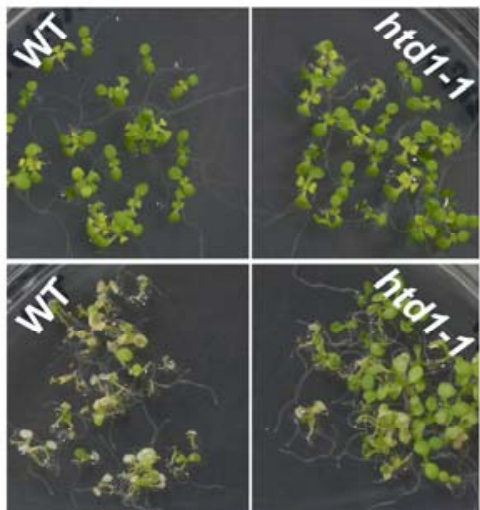

(-) Heat Shock

(+) Heat Shock
C

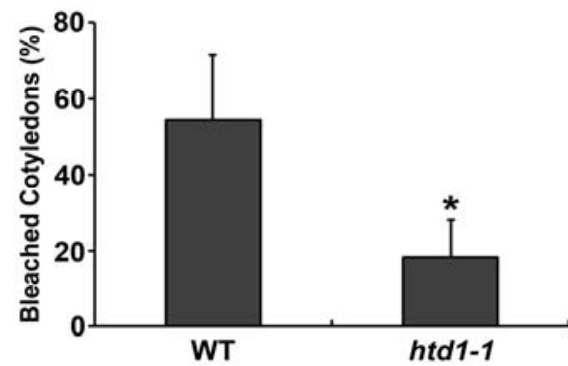

D

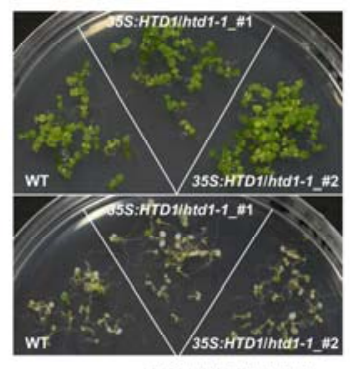

(-) Heat Shock

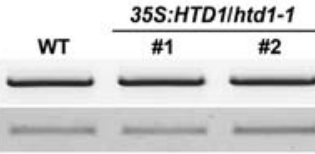

\section{(+) Heat Shock}

\section{HTD1}

RPN6a

Fig. 2. Correlation between HTD1 and heat stress phenotype. (A) Scheme of heat shock treatment in this study. (B) The phenotypes of wild type (WT) and $h t d 1-1$ plants in response to heat shock treatments. Three day-old wild type and $h t d 1-1$ seedlings grown at $22^{\circ} \mathrm{C}$ were incubated at $37^{\circ} \mathrm{C}$ for $3 \mathrm{~h}$ for acclimation, then allowed 3 days of recovery at $22^{\circ} \mathrm{C}$. Heat shock treatments at $45^{\circ} \mathrm{C}$ for 90 min were then applied, after which the seedlings were grown for an additional 3 days in order to monitor phenotypes. The plants were photographed 3 days after $45^{\circ} \mathrm{C}$ heat shock. (C) Rate of bleached cotyledons of wild type and $h t d 1-1$ seedlings under heat stress. Seedlings with more than $50 \%$ bleached cotyledons were counted from both samples. Values are the means $\pm S D(n=3)$. Statistically significant differences between the wild type and $h t d 1-$ 1 were identified by a Student's $t$-test; the single star indicates $P<0.05$. (D) The phenotypes of two HTD1-complementation lines in $h t d 1-1$ (35S:HTD1/htd1-1) in response to heat shock treatments. The condition for heat treatment was the same as that in (B). For semi-quantitative RT-PCR analysis to monitor HTD1 expressions, 9-day-old wild type and 35S:HTD1/htd1-1 seedlings grown in the absence of heat shock were used. Analysis was conducted with FP2 and RP2 primers. RPN6a was used as an internal control.

MATCHMAKER LexA Two-Hybrid System manual (Clontech). p80p-lacZ and the gene constructs cloned with pB42AD or pLexA were introduced into yeast strain EGY48. The transformants were then confirmed by growth on SD/-His/-Trp/-Ura plates. Interactions between proteins were monitored on SD/Gal/Raf/-His/Trp/-Ura plates including $80 \mathrm{mg} \mathrm{L}^{-1} \mathrm{X}$-Gal.

\section{In vivo co-immunoprecipitation assay}

Arabidopsis seedlings were resolved in protein extraction buffer [50 mM Tris- $\mathrm{HCl}$ (pH 7.5), $150 \mathrm{mM} \mathrm{NaCl}, 0.1 \%$ Tween 20, 10\% glycerol, $1 \mathrm{mM}$ DTT, $1 \mathrm{mM}$ phenylmethylsulfonyl fluoride and 1× complete protease inhibitor (Roche)]. Next $30-50 \mu \mathrm{l}$ of anti-MYC affinity matrix (COVANCE) were added to $1-5 \mathrm{mg}$ of protein extracts. The mixture was then incubated overnight at $4^{\circ} \mathrm{C}$ and washed at least three times with protein extraction buffers. For elution, beads with immunoprecipitated proteins were resuspended in Laemmli sample buffer (BioRad) and boiled for $5 \mathrm{~min}$.

\section{RESULTS AND DISCUSSION}

Identification of a possible CRL4 substrate receptor related to heat stress response

To determine if a fraction of CRL4 complexes participates in heat stress response, we monitored mRNA levels of 119 CRL4 substrate receptor genes (DWD and WDXR) with or without heat stress treatments using microarray data from the AtGenExpress Visualization Tool (AVT) (Kilian et al., 2007). Among them, one DWD gene (At2g19540) was upregulated by about 3.2 times in response to heat stress $\left(3.0 \mathrm{~h}\right.$ of $38^{\circ} \mathrm{C}$ heat stress followed by $1.0 \mathrm{~h}$ recovery at $25^{\circ} \mathrm{C}$ ). This gene was named HTD1. Our qPCR analysis confirmed that the transcript of HTD1 was increased by heat shock treatment, indicating possible involvement of HTD1 in cellular responses to heat stress (Fig. 3A). HTD1 is a 1407 bp region encoding 469 amino acids. A DWD domain of HTD1 is included in the WD40 domain region, similar to other DWD proteins (Figs. $1 \mathrm{~A}$ and $1 \mathrm{C}$ ). To investigate if the heat-inducibility of HTD1 is functionally related to heat stress response, its T-DNA insertion homozygous mutant, htd1-1 (SALK_081295), was obtained and analyzed for HTD1. T-DNA was inserted 279 bp upstream from the CDS start site of this gene (Fig. 1A). RT-PCR analysis showed that HTD1 expression was significantly decreased in htd1-1 (Fig. $1 \mathrm{~B}$ ), indicating that the insertion line could be useful for further study. We identified several HTD1 homologs from various plant species in the GenBank database, including Glycine max (soybean), Solanum lycopersicum (tomato), Oryza sativa (rice) and 
A

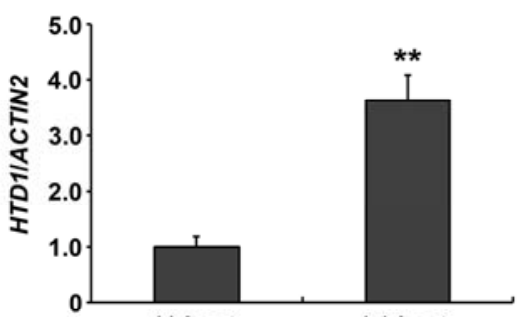

B

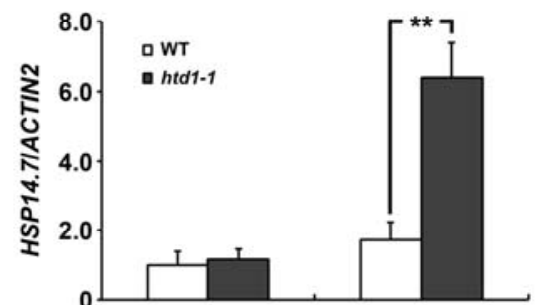

(-) heat

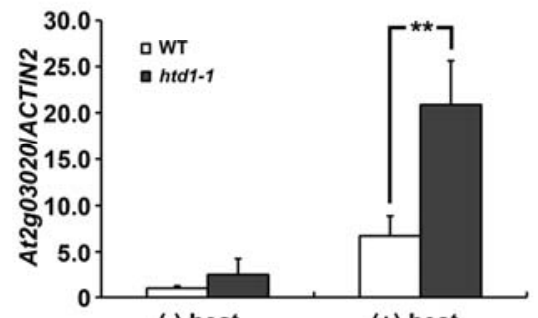

(-) heat
(+) heat

$(+)$ heat

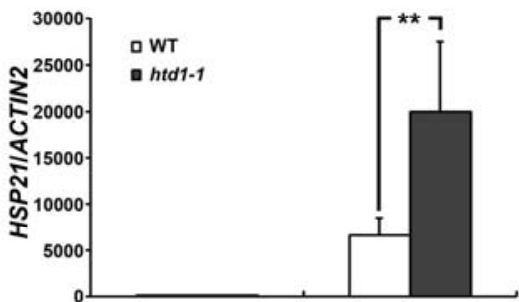

(-) heat

$(+)$ heat

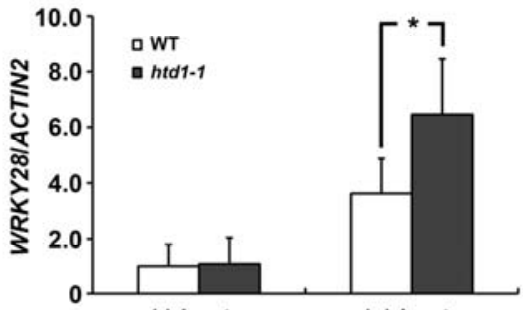

(-) heat
Fig. 3. Expression patterns of various heat-inducible genes in wild type and htd11. (A) Heat-inducibility of HTD1. Quantitative real-time PCR analysis was performed with wild type in the presence or absence of heat shock. The condition for heat treatment was the same as that in Fig. $2 \mathrm{~B}$. Relative amounts of HTD1 were normalized to the levels of ACTIN2 in the same sample. Values are the means $\pm \mathrm{SD}(\mathrm{n}=$ 3). Statistical analysis was conducted by a Student's t-test; ${ }^{* *} P<0.05$. (B) Hyperinduction of various heat-inducible genes in htd1-1. Quantitative real-time PCR analysis was performed with wild type and htd1-1 seedlings in the presence or absence of heat shock. The conditions for heat treatment were the same as described in Fig. 2B. Relative amounts of HSP14.7, HSP21, At2g03020 and WRKY28 were normalized to the levels of ACTIN2 in the same sample. Values are the means $\pm S D$ $(n=6)$. Statistical analysis was conducted by a Student's $t$-test; ${ }^{\star} P<0.05 ;{ }^{* \star} P<0.01$.
Zea mays (maize). All selected proteins exhibited high similarity (more than $74.9 \%$ ) at the amino acid level when compared to Arabidopsis HTD1 based on the EMBOSS Pairwise Alignment Algorithms at EMBL-EBI (http://www.ebi.ac.uk/Tools/emboss; Rice et al., 2000). All five proteins shared highly conserved WD40 and DWD regions and exactly matched the WDxR motif, which is a crucial site for binding to the adaptor protein of CRL4 complex (Fig. 1D). These results indicate that HTD1 and its related proteins may be highly conserved in the plant kingdom and play shared roles from dicots to monocots. Thus, it is expected that the results of the Arabidopsis HTD1 study could be applied to other useful crops such as rice, maize and tomato.

The decrease in HTD1 expression leads to heat stress tolerance

To elucidate the possible role of HTD1 in heat stress tolerance, three day-old wild type and $h t d 1-1$ seedlings were exposed to heat treatment at $37^{\circ} \mathrm{C}$ for $3 \mathrm{~h}$, followed by 3 days recovery time (for acclimation) before exposure to $45^{\circ} \mathrm{C}$ heat shock for 90 min. (Fig. 2A). Following heat shock, seedlings were grown for an additional 3 days to monitor phenotypic differences between wild type and $h t d 1-1$. Although a large portion of wild type seedlings collapsed and became bleached after heat shock, $h t d 1-1$ was much less affected by this treatment, indicating that it was thermotolerant (Fig. 2B). Indeed, the rate of bleached cotyledons was about three times lower in htd1-1 than the wild type (Fig. 2C). Next, to confirm whether the decrease in HTD1 expression was correlated with such tolerance phenotypes, we generated transgenic lines complementing htd1-1. Two independent complementation lines (35S:HTD1 in the background of $h t d 1-1$ ) showed thermotolerance levels similar to wild type (Fig. 2D). Therefore, the expression level of HTD1 is highly related to sensitivity to heat stress. Taken together, HTD1 functions as a negative regulator in thermotolerance in Arabidopsis. As shown in Fig. 1B, T-DNA insertion 279 bp upstream from the translation start site of HTD1 failed to knock-out HTD1 expression in $h t d 1-1$. Thus, the generation of transgenic lines that exhibit stronger repression of HTD1 expression would be required to generate plants with greater tolerance to heat stress, which would be useful in elucidation of the mechanism of HTD1 in heat stress response in plants in greater detail.

HTD1 negatively regulates the expression of various heatinducible genes

Since htd1-1 exhibited enhanced heat stress tolerance (Fig. 2), we investigated whether the decrease in HTD1 expression altered the expression pattern of heat-inducible genes. As shown in Fig. 3B, four heat-inducible genes, HSP14.7 (At5g47600), HSP21 (At4g27670), At2g03020 (as Heat shock protein HSP20/alpha crystallin family protein) and WRKY28 (At4g18170), were hyper-induced in response to heat stress, indicating that HTD1 could play a negative role in the expression of various heat-inducible genes. These results imply that the selected heat-stress inducible genes may contribute to enhancement of thermotolerance of $h t d 1-1$. Since small HSPs function as molecular chaperones that prevent misfolding and aggregation of 
A

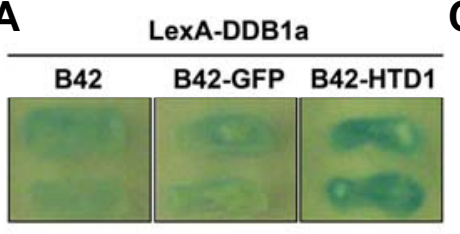

C LexA-HSP90-1

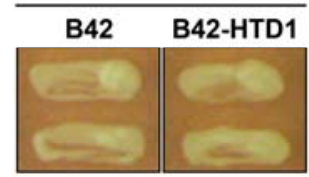

B

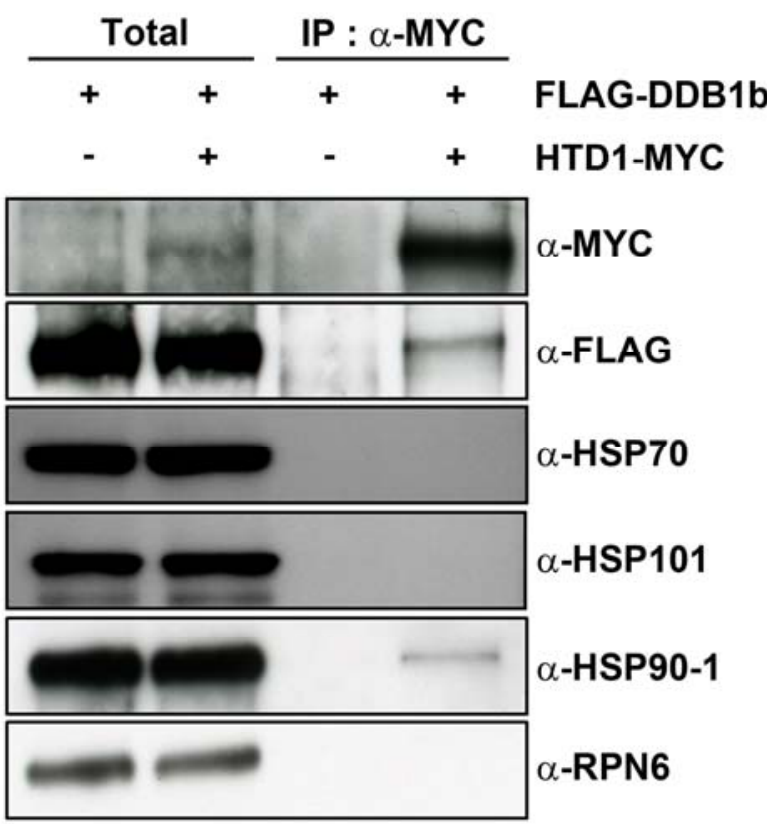

Fig. 4. Interaction patterns of various proteins with HTD1. (A) Direct interaction between HTD1 and the adaptor of the CRL4 complex based on yeast two-hybrid assays. Assays were performed with HTD1 protein as prey and DDB1a as bait to check their interactions. Empty vector (B42) and GFP proteins (B42-GFP) were used as negative controls. (B) Co-immunoprecipitation (Co-IP) assay of HTD1 with DDB1b and various HSP proteins. Transgenic plants overexpressing FLAG-DDB1b or FLAG-DDB1b/HTD1-MYC were used for these assays. The immunoblot used anti-RPN6 as a loading control. Total, $5 \%$ of the crude extracts used for Co-IP assays. (C) Interaction pattern between HTD1 and HSP90-1 in yeast two hybrid assay.

their client proteins caused by heat stress (Waters, 2013), the enhanced expression of our selected SHSP genes (HSP14.7, HSP21 and At2g03020) in htd1-1 suggest the involvement of genes in the heat tolerance of htd1-1. WRKY28 has been shown to participate in the activation of SA (salicylic acid) signal-related genes and defense responses against pathogens in Arabidopsis (van Verk et al., 2011). Additionally, when coexpressed with AtbHLH17, Arabidopsis WRKY28 is responsible for resistance against various abiotic stresses such as those caused by $\mathrm{NaCl}$, mannitol and oxidation, indicating its versatile role in plant biotic/abiotic stress responses (Babitha et al., 2013). Furthermore, several WRKY genes (WRKY25, WRKY 26 and $W R K Y$ 33) that show heat-inducibility have been reported to regulate the expression of heat-inducible genes in response to heat shock and to be responsible for thermotolerance (Li et al., 2011). Based on the above reports together with the heat-inducibility of WRKY28 (Fig. 3B), WRKY28 hyper-induced in htd1-1 might be involved in modulation of the thermotolerance process in Arabidopsis.

HTD1 may be a component of CRL4 complex

Since HTD1 possesses a putative DWD domain within WD40 repeats, we wondered if HTD1 could act as a part of CRL4 complexes. Therefore, we checked the interaction between DDB1a and HTD1 using a yeast two hybrid assay (Fig. 4A). Although the empty vector and green fluorescent protein (GFP) as prey showed weak binding activities with DDB1a as bait, HTD1 as prey exhibited much stronger binding activities with DDB1a than the above negative controls. To confirm that HTD1 is a component of the CRL4 complex in planta, we introduced HTD1-MYC constructs into FLAG-DDB1b/ddb1a lines to generate HTD1-MYC/FLAG-DDB1b/ddb1a plants, after which in vivo co-immunoprecipitation (co-IP) assays were performed to check the physical association between DDB1 and HTD1. As shown in Fig. 4B, MYC-tagged HTD1 proteins were successfully precipitated with FLAG-tagged DDB1b. Taken together, these results indicate that HTD1 interacts with the adaptor of the CRL4 complex and HTD1 may play its role as a possible component of CRL4 complex.

HTD1 can be associated with HSP90-1 in planta

As shown in Figs. 2 and 3, HTD1 negatively regulates the thermotolerance process and heat-induction of various heatinducible genes. For this action, HTD1 may directly interact with signaling component proteins involved in heat stress tolerance and negatively regulate their activities or stabilities since HTD1 is expected to function as the substrate receptor CRL4 E3 ligase (Figs. 4A and $4 \mathrm{~B}$ ). Three heat shock proteins were selected as the candidate substrates of CRL $4^{\mathrm{HTD} 1}$ in heat stress response, HSP70, HSP101 and HSP90-1 (an inducible form of HSP90), since they play a central role in thermotolerance in plants (Wang et al., 2004). To check whether CRL4 ${ }^{\mathrm{HTD} 1}$ is directly involved in the decrease of activities or stabilities of such HSP proteins via UPS (ubiquitin-proteasome system), we investigated the in vivo association pattern between HTD1 and the HSPs. The co-IP assays demonstrated that HTD1 was associated with HSP90-1, but not with HSP70 and HSP101, in planta (Fig. 4B), implying the functional connection between HSP90-1 and HTD1. However, in our yeast two hybrid assay, HSP90-1 did not directly interact with HTD1 (Fig. 4C). Therefore, it is thought that the negative regulation of HTD1 in thermotolerance may be related to the indirect physical association between HTD1 and HSP90-1, at least in part, and that this association seems to require the mediation of an unknown factor. Based on this association, we hypothesized that HTD1 could be involved in degradation of HSP90-1. Actually, it was reported that taxotere (a cancer chemotherapeutic drug) reduced the function of HSP9O by ubiquitination and subsequent proteasomal degradation of HSP90 in HUVEC, indicating that HSP90 could be a target of ubiquitylation and degraded via UPS (Murtagh et al., 2006). However, when we compared HSP90-1 protein levels following heat stress in wild type and $h t d 1-1$, the levels of HSP90-1 proteins from both plants were comparable (Supplementary Fig. S1). These results indicate that the CRL4 complex mediated by HTD1 might play a different role from a typical E3 ubiquitin ligase complex, which is responsible for the substrate recognition and degradation via UPS. It was recently reported that REPRESSOR OF UV-B PHOTOMORPHOGENESIS 1 (RUP1) and RUP2 function as negative regulators in both processes of photomorphogenesis 
and stress acclimation mediated by UV-B in Arabidopsis (Gruber et al., 2010). These genes commonly possess the conserved DWD domains and have therefore been categorized into the Arabidopsis DWD protein group (Lee et al., 2008), despite lack of evidence of their direct interactions with DDB1. They inhibit the interaction between UV RESISTANCE LOCUS 8 (UVR8) and CONSTITUTIVELY PHOTOMORPHOGENIC 1 (COP1) via competition with COP1 for binding of UVR8, which is essential for the UV-B signal transduction pathway. This competition leads to facilitation of UVR8 redimerization, which makes UVR8 inactive. This report implies that a portion of the proteins categorized into the DWD group play an atypical role rather than the role as E3 ligase for substrate destruction. Arabidopsis ROF1 (AtFKBP62) has been reported to associate with HSP90-1-HsfA2 and play a role in long term acquired thermotolerance by modulating the expression levels of HsfA2dependent SHSPs, which are responsible for heat stress tolerance (Meiri and Breiman, 2009), whereas ROF2 is a ROF1 homologous protein that interacts directly with ROF1 and negatively functions in expression of SHSPs by joining in the ROF1HSP90-1-HsfA2 complex and sequestering ROF1 from the complex during recovery from heat stress (Meiri et al., 2010). Similar to ROF2, the possible function of association/sequestration of HTD1 might be to disturb the role of a co-chaperone (like ROF1) that helps HSP90-1 function, which would explain the connection between HTD1/HSP90-1 association and the hyper-induction of SHSPs in htd1-1 observed in our study (Figs. $3 B$ and $4 B$ ). Alternatively, HTD1 might interfere with the action of HSP90-1 in the heat tolerance process by associating with HSP90-1 via an unknown adaptor that links between HTD1 and HSP90-1, and then keeping HSP90-1 away from the formation of multichaperone complexes. However, we cannot exclude the possibility that HSP90-1 can be monoubiquitinated by CRL $4^{\mathrm{HTD} 1}$ which leads to the alteration of HSP90-1's activity independent of proteolysis. Therefore, further investigation of the ubiquitination pattern of HSP90-1 under the presence/absence of HTD1 would be required for the detailed elucidation of the relationship between HSP90-1 and HTD1. Moreover, since it may be possible that CRL4 ${ }^{\mathrm{HTD} 1}$ negatively regulates the stability of unidentified positive regulator(s) in heat stress signaling, additional investigations of the binding partners of HTD1 as potential substrates should be conducted to determine if a possible CRL4 complex mediated by HTD1 could exert E3 ligase activity for protein degradation.

Here, we report a new type of DWD protein that negatively regulates the thermotolerance process in Arabidopsis. Although a decrease of HTD1 expression results in enhanced thermotolerance and increased expression of various heatinducible genes, detailed information regarding the role of HTD1 in heat stress response was not fully provided by this study. Further evidence of the role of HTD1 in the thermotolerance process could be obtained by genetic combination between $h t d 1-1$ and various heat stress-related mutants such as rof1 and knockout mutants related to HSP and Hsf genes. Furthermore, based on the link between HTD1 and HSP90 observed in the present study and reports of the versatile role of HSP90 involved in biotic and abiotic stresses (Botër et al., 2007; Krishna and Gloor, 2001), investigation of the potential role of HTD1 in other stress signaling pathways would serve as a platform for elucidation of the detailed action mechanism of HTD1 in plants.

Note: Supplementary information is available on the Molecules and Cells website (uww.molcells.org).

\section{ACKNOWLEDGMENTS}

This research was supported by the Basic Science Research Program through the National Research Foundation of Korea (NRF) funded by the Ministry of Education, Science and Technology (NRF-2012R1A1A1001564), by iPET (Korea Institute of Planning and Evaluation for Technology in Food, Agriculture, Forestry and Fisheries), Ministry of Agriculture, Food and Rural Affairs (112030-1), and by a grant from the Next-Generation BioGreen 21 Program (No. PJ009010), Rural Development Administration, Republic of Korea to J.-H.L. This work was also supported by grants from National Basic Research Program of China (973 Program) (2012CB910900) and NIH (GM-47850) to X.W.D.

\section{REFERENCES}

Angers, S., Li, T., Yi, X., MacCoss, M.J., Moon, R.T., and Zheng, N. (2006). Molecular architecture and assembly of the DDB1CUL4A ubiquitin ligase machinery. Nature 443, 590-593.

Babitha, K.C., Ramu, S.V., Pruthvi, V., Mahesh, P., Nataraja, K.N. and Udayakumar, M. (2013). Co-expression of AtbHLH17 and AtWRKY28 confers resistance to abiotic stress in Arabidopsis. Transgenic Res. 22, 327-341.

Baniwal, S.K., Bharti, K., Chan, K.Y., Fauth, M., Ganguli, A., Kotak, S., Mishra, S.K., Nover, L., Port, M., Scharf, K.D., et al. (2004). Heat stress response in plants: a complex game with chaperones and more than twenty heat stress transcription factors. J. Biosci. 29, 471-487.

Biedermann, S., and Hellmann, H. (2010). The DDB1a interacting proteins ATCSA-1 and DDB2 are critical factors for UV-B tolerance and genomic integrity in Arabidopsis thaliana. Plant J. 62, 404-415.

Bohnert, H.J., Gong, Q., Li, P., and Ma, S. (2006). Unraveling abiotic stress tolerance mechanisms - Getting genomics going. Curr. Opin. Plant Biol. 9, 180-188.

Boston, R.S., Viitanen, P.V., and Vierling, E. (1996). Molecular chaperones and protein folding in plants. Plant Mol. Biol. 32, 191-222.

Botër, M., Amigues, B., Peart, J., Breuer, C., Kadota, Y., Casais, C., Moore, G., Kleanthous, C., Ochsenbein, F., Shirasu, K., and Guerois, R. (2007). Structural and functional analysis of SGT1 reveals that its interaction with HSP90 is required for the accumulation of $\mathrm{Rx}$, an R protein involved in plant immunity. Plant Cell 19, 3791-3804.

Chen, H., Shen, Y., Tang, X., Yu, L., Wang, J., Guo, L., Zhang, Y., Zhang, H., Feng, S., Strickland, E., et al. (2006). Arabidopsis CULLIN4 forms an E3 ubiquitin ligase with RBX1 and the CDD complex in mediating light control of development. Plant Cell 18, 1991-2004.

Clough, S.J., and Bent, A.F. (1998). Floral dip: a simplified method for Agrobacterium-mediated transformation of Arabidopsis thaliana. Plant J. 16, 735-743.

Dreher, K., and Callis, J. (2007). Ubiquitin, hormones and biotic stress in plants. Ann. Bot. 99, 787-822.

Gruber, H., Heijde, M., Heller, W., Albert, A., Seidlitz, H.K., and Ulm, R. (2010). Negative feedback regulation of UV-B-induced photomorphogenesis and stress acclimation in Arabidopsis. Proc. Natl. Acad. Sci. USA 107, 20132-20137.

He, Y.J., McCall, C.M., Hu, J., Zeng, Y., and Xiong, Y. (2006). DDB1 functions as a linker to recruit receptor WD40 proteins to CUL4-ROC1 ubiquitin ligases. Genes Dev. 20, 2949-2954.

Hershko, A., and Ciechanover, A. (1998). The ubiquitin system. Annu. Rev. Biochem. 67, 425-479.

Higa, L.A., Wu, M., Ye, T., Kobayashi, R., Sun, H., and Zhang, H. (2006). CUL4-DDB1 ubiquitin ligase interacts with multiple WD40-repeat proteins and regulates histone methylation. Nat. Cell Biol. 8, 1277-1283.

Hong, S.W., and Vierling, E. (2001). Hsp101 is necessary for heat tolerance but dispensable for development and germination in the absence of stress. Plant J. 27, 25-35.

Jin, J., Arias, E.E., Chen, J., Harper, J.W., and Walter, J.C. (2006). A family of diverse Cul4-Ddb1-interacting proteins includes Cdt2, which is required for $\mathrm{S}$ phase destruction of the replication factor 
Cdt1. Mol. Cell 23, 709-721

Kampinga, H.H., Brunsting, J.F., Stege, G.J., Burgman, P.W., and Konings, A.W. (1995). Thermal protein denaturation and protein aggregation in cells made thermotolerant by various chemicals: role of heat shock proteins. Exp. Cell Res. 219, 536-546.

Kilian, J., Whitehead, D., Horak, J., Wanke, D., Weinl, S., Batistic, O., D'Angelo, C., Bornberg-Bauer, E., Kudla, J., and Harter, K (2007). The AtGenExpress global stress expression data set: protocols, evaluation and model data analysis of UV-B light, drought and cold stress responses. Plant J. 50, 347-363.

Krishna, P., and Gloor, G. (2001). The Hsp90 family of proteins in Arabidopsis thaliana. Cell Stress Chaperones 6, 238-246.

Lee, J.H., and Schöffl, F. (1996). An Hsp70 antisense gene affects the expression of $\mathrm{HSP} 70 / \mathrm{HSC} 70$, the regulation of HSF and the acquisition of thermotolerance in transgenic Arabidopsis thaliana. Mol. Gen. Genet. 252, 11-19.

Lee, U., Wie, C., Escobar, M., Williams, B., Hong, S.W., and Vierling, E. (2005). Genetic analysis reveals domain interactions of Arabidopsis Hsp100/ClpB and cooperation with the small heat shock protein chaperone system. Plant Cell 17, 559-571.

Lee, J.H., Terzaghi, W., Gusmaroli, G., Charron, J.B., Yoon, H.J., Chen, H., He, Y.J., Xiong, Y., and Deng, X.W. (2008). Characterization of Arabidopsis and rice DWD proteins and their roles as substrate receptors for CUL4-RING E3 ubiquitin ligases. Plant Cell 20, 152-167.

Lee, J.H., Yoon, H.J., Terzaghi, W., Martinez, C., Dai, M., Li, J., Byun, M.O., and Deng, X.W. (2010). DWA1 and DWA2, two Arabidopsis DWD protein components of CUL4-based E3 ligases, act together as negative regulators in $\mathrm{ABA}$ signal transduction. Plant Cell 22, 1716-1732.

Li, S., Fu, Q., Chen, L., Huang, W., and Yu, D. (2011). Arabidopsis thaliana WRKY25, WRKY26, and WRKY33 coordinate induction of plant thermotolerance. Planta 233, 1237-1252.

Meiri, D., and Breiman, A. (2009). Arabidopsis ROF1 (FKBP62) modulates thermotolerance by interacting with HSP90.1 and affecting the accumulation of HsfA2-regulated sHSPs. Plant J. 59, 387-399.

Meiri, D., Tazat, K., Cohen-Peer, R., Farchi-Pisanty, O., AviezerHagai, K., Avni, A., and Breiman, A. (2010). Involvement of Arabidopsis ROF2 (FKBP65) in thermotolerance. Plant Mol. Biol. 72 , 191-203.

Mittler, R., and Blumwald, E. (2010). Genetic engineering for modern agriculture: challenges and perspectives. Annu. Rev. Plant Biol. 61, 443-462.

Mittler, R., Finka, A., and Goloubinoff, P. (2012). How do plants feel the heat? Trends Biochem. Sci. 37, 118-125.

Murtagh, J., Lu, H., and Schwartz, E.L. (2006). Taxotere-induced inhibition of human endothelial cell migration is a result of heat shock protein 90 degradation. Cancer Res. 66, 8192-8199.

Neuwald, A.F., Aravind, L., Spouge, J.L., and Koonin, E.V. (1999). AAAt: A class of chaperone-like ATPases associated with the assembly, operation, and disassembly of protein complexes. Genome Res. 9, 27-43.

Ono, K., Hibino, T., Kohinata, T., Suzuki, S., Tanaka, Y., Nakamura, T., Takabe, T., and Takabe, T. (2001). Overexpression of DnaK from a halotolerant cyanobacterium Aphanothece halophytica enhances the high temperature tolerance of tobacco during germination and early growth. Plant Sci. 160, 455-461.
Rice, P., Longden, I., and Bleasby, A. (2000). EMBOSS: the European Molecular Biology Open Software Suite. Trends Genet. 16, 276-277.

Richter, K., and Buchner, J. (2001). Hsp90: chaperoning signal transduction. J. Cell. Physiol. 188, 281-290.

Ruelland, E., and Zachowski, A. (2010). How plants sense temperature. Environ. Exp. Bot. 69, 225-232.

Schramm, F., Ganguli, A., Kiehlmann, E., Englich, G., Walch, D., and von Koskull-Döring, P. (2006). The heat stress transcription factor HsfA2 serves as a regulatory amplifier of a subset of genes in the heat stress response in Arabidopsis. Plant Mol. Biol. 60, 759-772.

Seo, K.I., Lee, J.H., Nezames, C.D., Zhong, S., Song, E., Byun, M.O., and Deng, X.W. (2014). ABD1 is an Arabidopsis DCAF substrate receptor for CUL4-DDB1-based E3 ligases that acts as a negative regulator of abscisic acid signaling. Plant Cell 26 , 695-711.

Smalle, J., and Vierstra, R.D. (2004). The ubiquitin 26 S proteasome proteolytic pathway. Annu. Rev. Plant Biol. 55, 555-590.

Sugino, M., Hibino, T., Tanaka, Y., Nii, N., Takabe, T., and Takabe, T. (1999). Overexpression of DnaK from a halotolerant cyanobacterium Aphanothece halophytica acquires resistance to salt stress in transgenic tobacco plants. Plant Sci. 146, 81-88.

Sung, D.Y., Vierling, E., and Guy, C.L. (2001). Comprehensive expression profile analysis of the Arabidopsis Hsp70 gene family Plant Physiol. 126, 789-800.

Todaka, D., Nakashima, K., Shinozaki, K., and YamaguchiShinozaki, K. (2012). Toward understanding transcriptional regulatory networks in abiotic stress responses and tolerance in rice. Rice 5, 6 .

van Verk, M.C., Bol, J.F., and Linthorst, H.J. (2011). WRKY transcription factors involved in activation of SA biosynthesis genes. BMC Plant Biol. 11, 89.

Vierling, E. (1991). The roles of heat shock proteins in plants. Annu. Rev. Plant Physiol. Plant Mol. Biol. 42, 579-620.

Vierstra, R.D. (2009). The ubiquitin-26S proteasome system at the nexus of plant biology. Nat. Rev. Mol. Cell Biol. 10, 385-397.

von Koskull-Döring, P., Scharf, K.D., and Nover, L. (2007). The diversity of plant heat stress transcription factors. Trends Plant Sci. 12, 452-457.

Wang, W., Vinocur, B., Shoseyov, O., and Altman, A. (2004). Role of plant heat-shock proteins and molecular chaperones in the abiotic stress response. Trends Plant Sci. 9, 244-252.

Waters, E.R. (2013) The evolution, function, structure, and expression of the plant SHSPs. J. Exp. Bot. 64, 391-403.

Yabe, N., Takahashi, T., and Komeda, Y. (1994). Analysis of tissuespecific expression of Arabidopsis thaliana Hsp90-family gene HSP81. Plant Cell Physiol. 35, 1207-1219.

Zhang, Y., Feng, S., Chen, F., Chen, H., Wang, J., McCall, C., Xiong, Y., and Deng, X.W. (2008). Arabidopsis DDB1-CUL4 ASSOCIATED FACTOR1 forms a nuclear E3 ubiquitin ligase with DDB1 and CUL4 that is involved in multiple plant developmental processes. Plant Cell 20, 1437-1455.

Zhang, C., Guo, H., Zhang, J., Guo, G., Schumaker, K.S., and Guo, Y. (2010). Arabidopsis cockayne syndrome A-like proteins $1 \mathrm{~A}$ and $1 \mathrm{~B}$ form a complex with CULLIN4 and damage DNA binding protein $1 \mathrm{~A}$ and regulate the response to UV irradiation. Plant Cell 22, 2353-2369. 